\title{
Influence analysis of beam support mode on linear guide deformation
}

\author{
Jiangping $\mathrm{Cao}^{1}$, Gang $\mathrm{Li}^{2}{ }^{2}{ }^{*}$, Yuanhui Peng ${ }^{2}$, and Chang Yang ${ }^{1}$ \\ ${ }^{1}$ National Institute of Measurement and Testing Technology, China \\ ${ }^{2}$ Sichuan province in the measuring instrument technology co., LTD Cheng Du, China
}

\begin{abstract}
As the basis of carrying guide rail operation, beam support mode has been widely used in high-end manufacturing, equipment, mechanical engineering and other fields. With beam support and rotating structure driving position and attitude changes, flexible three-dimensional space movement can be realized.The key technology of the beam support to drive the branch guide rail is to control the deformation of the guide rail. The deformation of the guide rail mainly affects the guide rail structure, stiffness, supporting mode, driver design and load, etc.When the driving mode, driving position and driving force, guide bracket (including bracket and connection mode) and load (including mass load, load moving position and moving range) are determined,Or affected by the use of the requirements of the adjustment range is very small, the beam support mode becomes the main influencing factor of guide deformation, especially in the rotary state of the branch guide, guide force, deformation, deflection and so on will show different trends, such as the maximum deformation of the driving end may be 26 times.This paper through the analysis of the main parameters affecting the guide rail deformation characteristics, the default part, on the basis of mathematical modeling and simulation analysis with Solidworks software, compare a variety of support and attitude under the guide of stress and deformation, gives the relative deformation degree under different mode, can provide a reference for relevant involved.
\end{abstract}

\section{Introduction}

Rotating beam support and linear guide ways can implement space flexible $3 \mathrm{~d}$ motion and attitude change, for in a narrow space structure is compact, and needs to have the place of reciprocating motion, through the adjustment and design support structure, load and the driving mode, etc., can be realized under the condition of high load to ensure the space of high precision linear motion.In practical engineering application, the beam supports the guide rail as the main component of supporting and guiding motion in the linear guide rail.The key technology of the beam support to drive the branch guide rail is to control the deformation of the guide rail. The deformation of the guide rail mainly affects the guide rail structure, stiffness, supporting mode, driver design and load, etc.When the driving mode, driving position and driving force, guide bracket (including bracket and connection mode)

\footnotetext{
Corresponding author: 67732004@qq.com
} 
and load (including mass load, load moving position and moving range) are determined,Or affected by the use of the requirements of the adjustment range is very small, the beam support mode becomes the main factor affecting the deformation of the guide rail, especially in the rotation of the branch guide rail, guide force, deformation, deflection and so on will show different trends[1]. The mathematical model is established according to the requirements, as shown in Figure 1: the structure layout mode shown in the simulation model of multi-attitude guide rail. The stress on the guide rail, the micro-displacement (deformation) of the guide rail and the trend of change with time are all different.By default part this paper, on the basis of mathematical modeling and simulation analysis with Solidworks software, compare a variety of the stress and deformation of the support and attitude under the guide, to discuss in the drive and load conditions, different forms of support for linear guide pair of deformation and the influence of changing trends, to provide the reference for related design work [2].

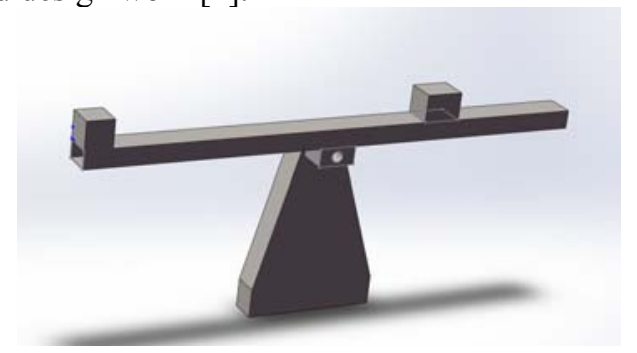

Fig. 1. More attitude guide simulation model.

\section{Support structure and the impact analysis}

Gesture more linear guide bracket structure including stents way, posture and load structure.In the three-dimensional space state against doing more straight line reciprocating movement posture guide rail, drive weight and load requirements are lightweight design, in order to reduce the deformation and gravity, and compact structure as far as possible, as far as possible short, heavy torque after ensure that these two points, in order to achieve more posture, reduce interference in the posture change, selection and design of support way is particularly important, different ways of stents, may cause big differences in the overall device will influence.In this paper, the movement process of the gesture more guide rail caused deformation, analyzing the structure of the problem of choosing guide three kinds of typical working condition corresponding to three kinds of typical framework model, discussed under the condition of default, the nine kinds of deformation model of guide rail[3].

First of all, many gestures guide of typical working condition including horizontal, inclined to $30^{\circ}, 60^{\circ}$ oblique position and vertical, due to the vertical position of longitudinal straight line guide rail, less affected by gravity, so here to choose the other three states are analyzed.On both ends of the guide bracket structure contains, among, and between the two of three points location, figure 1 model is the intermediate support way.Then, determine the preset conditions, select the $2.7 \mathrm{~m}$ guide bracket test analysis, load $4 \mathrm{~kg}$, at $1.9 \mathrm{~m}$ distance drive, drive $3.5 \mathrm{~kg}$, placed in the guide rail bracket at the left.Mathematical model is set up in Solidworks and implanted into the preset conditions, as shown in figure a, build different profile with different support model, and then to choose the appropriate parameter setting in Solidworks Simulation plug-in connection, contact, fixed, load (gravity), through the analysis of parsing guide force and deformation.Below is for the three position corresponding to three kinds of framework mode for specific analysis[4]. 


\subsection{Horizontal posture mode}

In the established mathematical model of Solidworks, design parameters, construction of horizontal posture.Designed to build support among model in Solidworks Simulation plugin set connection, contact, fixed, load (gravity), analytical operation guide force and deformation condition, the following figure 2: horizontal posture with intermediate support patterns shown in deformation analysis, linear guide rail pair maximum deformation occurs at the drive end, on the other side also have deformation, but relatively small.Design support patterns on both ends of the building, in Solidworks Simulation plug-in set connection, contact, fixed, load (gravity), parsing operation guide force and deformation condition, the following figure 3: horizontal posture with two-terminal bracing pattern shown in deformation analysis, linear guide rail pair maximum deformation occurred in the middle.Design build three points location support model in Solidworks Simulation plug-in set connection, contact, fixed, load (gravity), parsing operation guide force and deformation condition, the following figure 4: horizontal posture with three location support patterns shown in deformation analysis, linear guide maximum deformation occurs mainly at the drive end position. [5]

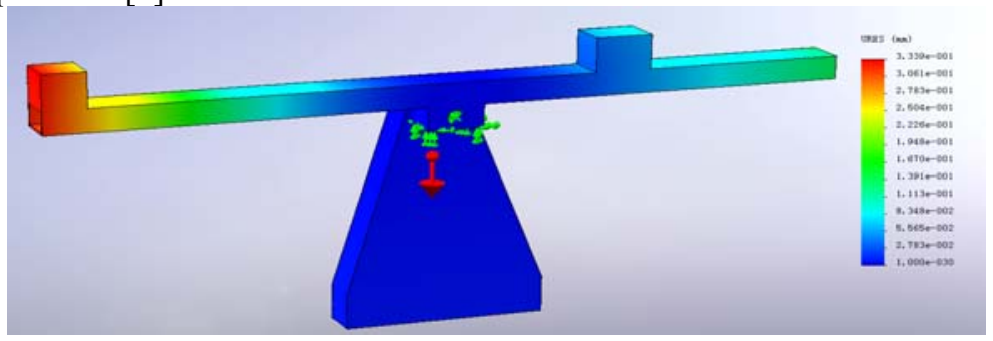

Fig. 2. Horizontal posture with intermediate support deformation analysis model.

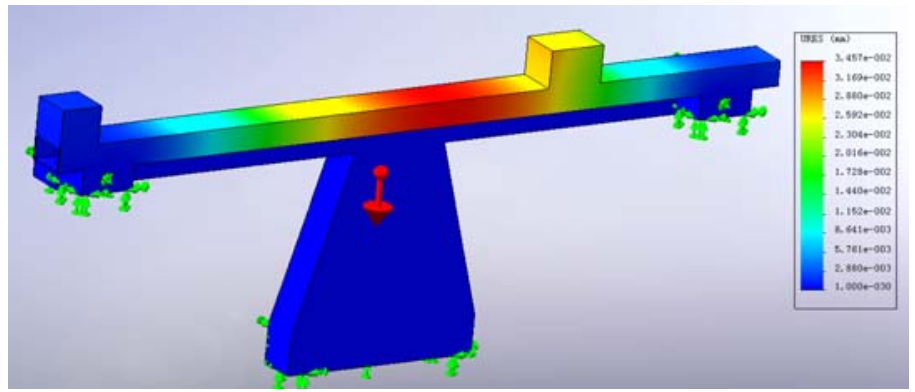

Fig. 3. The horizontal posture with two-terminal bracing pattern deformation analysis diagram.

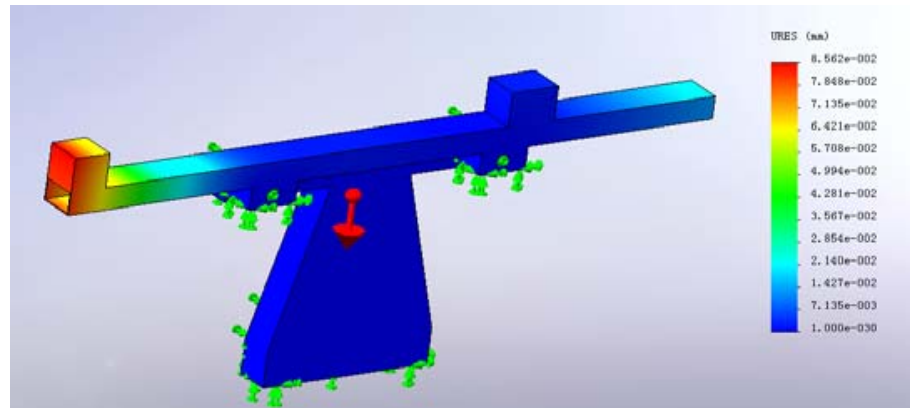

Fig. 4. Horizontal posture support deformation analysis model with three position. 
By way of comparative analysis in the three levels of state support deformation state, found that the middle is

supported on both ends of the maximum deformation of about ten times the maximum deformation, three position is supported on both ends of the maximum deformation of about twice the maximum deformation, static level state, in the same conditions on both ends of the support deformation smaller, support rigidity is better.

\section{$2.230^{\circ}$ oblique position mode}

In the established mathematical model of Solidworks, redesigned to adjust parameters, to build $30^{\circ}$ oblique gesture.Designed to build support among model in Solidworks Simulation plug-in set connection, contact, fixed, load (gravity), analytical operation guide force and deformation condition, the following figure 5:30 ${ }^{\circ}$ oblique gesture with intermediate support patterns shown in deformation analysis, linear guide both ends have deformation, in comparison, the drive end deformation is bigger, drive on deformation of the maximum deformation at the other end of a times the left and right sides.Design support patterns on both ends of the building, in Solidworks Simulation plug-in set connection, contact, fixed, load (gravity), parsing operation guide force and deformation condition, the following figure 6:30 ${ }^{\circ}$ oblique gesture with two-terminal bracing pattern shown in deformation analysis, linear guide maximum deformation occurred in the middle.Design build three points location support model in Solidworks Simulation plug-in set connection, contact, fixed, load (gravity), parsing operation guide force and deformation condition, the following figure 7:30 ${ }^{\circ}$ oblique stance with three location support patterns shown in deformation analysis, linear guide both ends have deformation, maximum deformation occurred in the drive end position, drive maximum deformation is about four times that of the other end of the maximum deformation.

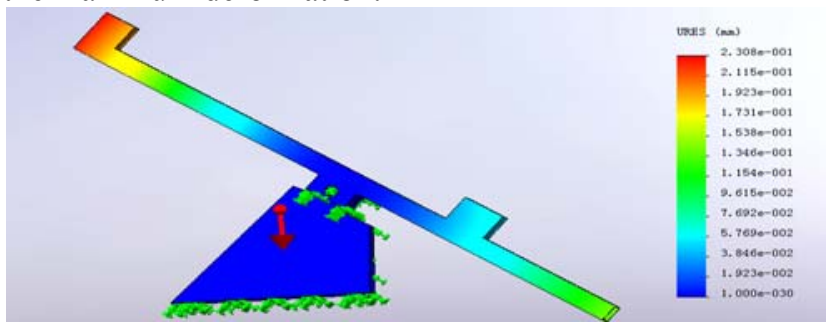

Fig. 5. Among $30^{\circ}$ oblique gesture with support deformation analysis model.

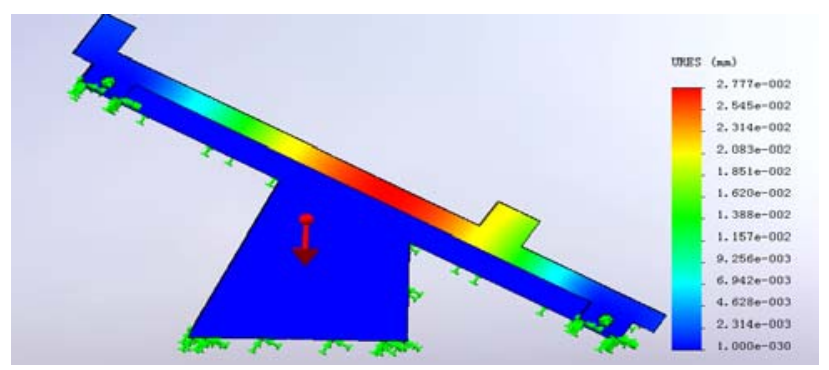

Fig. 6. $30^{\circ}$ oblique position with two-terminal bracing pattern deformation analysis diagram. 


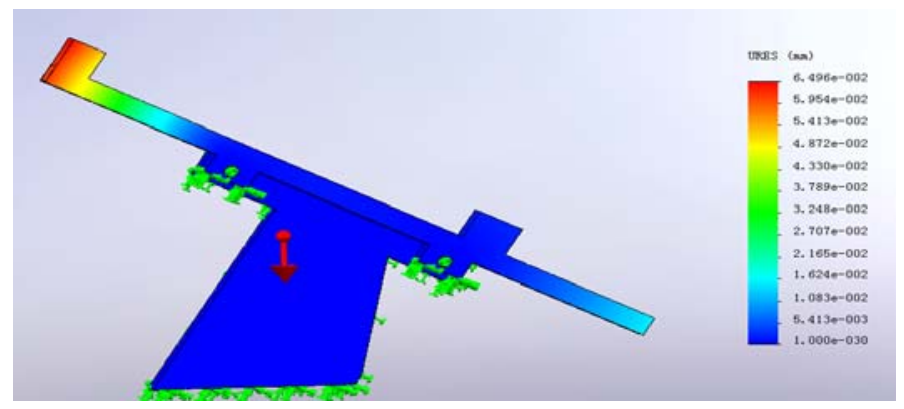

Fig. 7. $30^{\circ}$ oblique gesture support deformation analysis model with three position.

Clear from the above analysis diagram, three ways of distortion, location and extent have intermediate support mode maximum deformation occurs at the drive end, maximum $2.308 \mathrm{e}-001 \mathrm{~mm}$, two-terminal bracing pattern maximum deformation occurred in the middle position, the largest $2.777 \mathrm{e}-002 \mathrm{~mm}$, three points location maximum deformation occurs at the drive end support mode, maximum $6.496 \mathrm{e}-002 \mathrm{~mm}$, is found that the middle support patterns supported on both ends of the maximum deformation of about 8 times, three points location support patterns supported on both ends of the maximum deformation is about 2 times, you can see, under this kind of posture, linear guide bracket on both ends of the support mode of deformation smaller, better rigidity and stability[6].

\subsection{Oblique $60^{\circ}$ gesture mode}

In the established mathematical model of Solidworks, to design parameters, construction of oblique $60^{\circ}$.Designed to build support among model in Solidworks Simulation plug-in set connection, contact, fixed, load (gravity), analytical operation guide force and deformation condition, the following figure 8: oblique $60^{\circ}$ gesture with intermediate support patterns shown in deformation analysis diagram, there are deformation on both ends of the linear guide, in comparison, the drive end deformation smaller than the other end, drive the largest deformation about e-002 in $6.806 \mathrm{~mm}$, the other end of the maximum deformation about e002 in $9.075 \mathrm{~mm}$, about 1.3 times.Design support patterns on both ends of the building, parsing operation guide force and deformation condition, the following figure 9: oblique $60^{\circ}$ gesture with two-terminal bracing pattern shown in deformation analysis, linear guide maximum deformation occurred in the middle. Design to build three points location support model, analytical operation guide force and deformation condition, the following figure 7 : oblique $60^{\circ}$ gesture with three position support patterns shown in deformation analysis, linear guide both ends have deformation, maximum deformation occurred in the drive end position, drive maximum deformation is about three times as much as the other end of the maximum deformation.

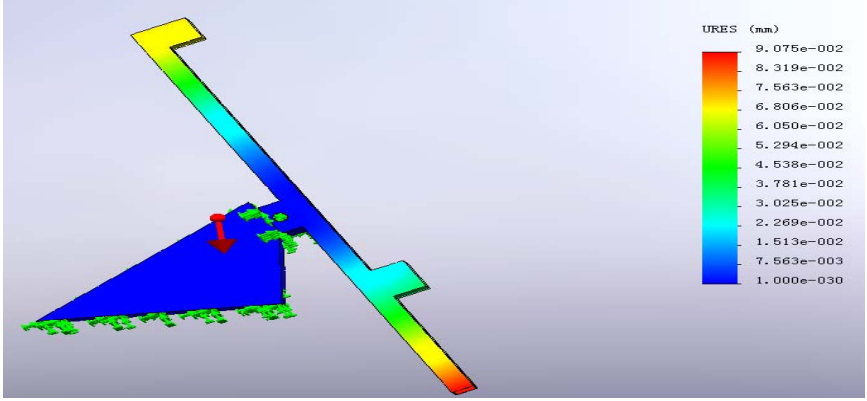

Fig. 8. Inclined to $60^{\circ}$ gesture with intermediate support deformation analysis model. 


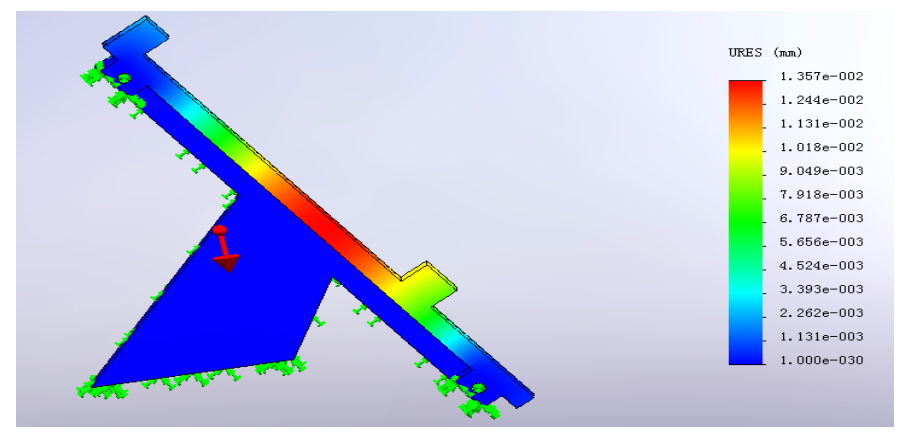

Fig. 9. Inclined to $60^{\circ}$ gesture with two-terminal bracing pattern deformation analysis diagram.

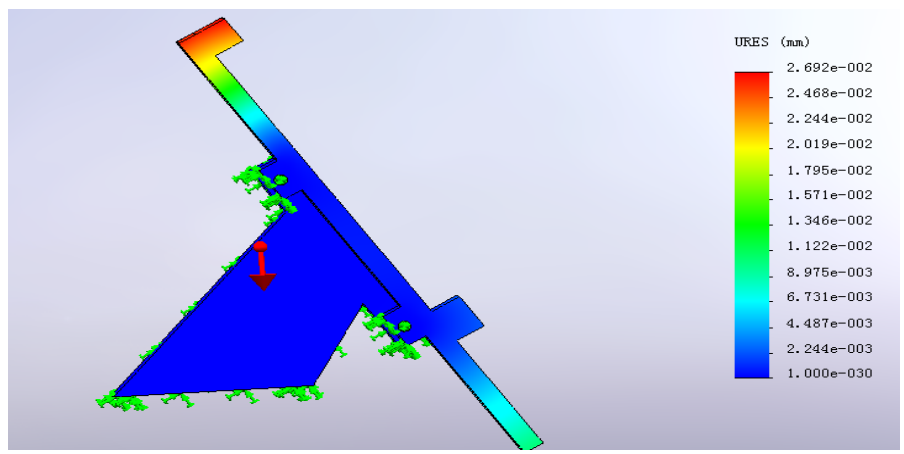

Fig. 10. Inclined to $60^{\circ}$ gesture support deformation analysis model with three position.

Clear from the above analysis diagram, three ways of distortion, location and extent have intermediate support mode maximum deformation occurred on the drive end at the other end, the largest $9.075 \mathrm{e}-002 \mathrm{~mm}$, two-terminal bracing pattern maximum deformation occurred in the middle position, the largest $1.357 \mathrm{e}-002 \mathrm{~mm}$, three points location maximum deformation occurs at the drive end support mode, maximum $2.692 \mathrm{e}-002 \mathrm{~mm}$, is found that the middle support patterns supported on both ends of the maximum deformation of about 6 times, three points location support patterns supported on both ends of the maximum deformation is about 2 times, you can see, under this kind of posture, linear guide bracket on both ends of the support mode of deformation smaller, better rigidity and stability.

\subsection{Comprehensive analysis}

Nine kinds of model construction from the above comprehensive analysis and comparison of simulation data of maximum deformation (see table 1: maximum deformation analysis table), you can see that under the same gesture, maximum deformation minimum on both ends of the supporting way, and with the increase of attitude Angle, three kinds of support way cause the biggest deformation decrease gradually.

Table 1. The biggest deformation analysis table.

\begin{tabular}{|c|c|c|c|}
\hline & The middle support & $\begin{array}{c}\text { On both ends of the } \\
\text { support }\end{array}$ & $\begin{array}{c}\text { "П type support by one- } \\
\text { third }\end{array}$ \\
\hline level & $3.339 \mathrm{e}-001$ & $3.457 \mathrm{e}-002$ & $8.562 \mathrm{e}-002$ \\
\hline $30^{\circ}$ inclined & $2.308 \mathrm{e}-001$ & $2.777 \mathrm{e}-002$ & $6.496 \mathrm{e}-002$ \\
\hline $60^{\circ}$ inclined & $9.075 \mathrm{e}-002$ & $1.357 \mathrm{e}-002$ & $2.692 \mathrm{e}-002$ \\
\hline
\end{tabular}


Can be seen from the above analysis, in a relatively short stroke cases in the process of using multiple posture guide, the smaller the farther support spacing of rail deformation, tend to use gesture month vertical position, the smaller the deformation, therefore, in many gesture guide the design and use process, in the case of position structure allows, especially under the condition of considering the influences of drive and load bearing is required, priority on both ends of the support way, framework and location to combine driving gear, bearing, and guide structure;At the same time, the more you try to make in use and downtime linear guide in a larger Angle, had better be upright posture, to ensure the stability and accuracy of attitude more guide.

\section{Conclusion}

In the beam supporting drive linear guide movement of the structure, especially the presence of rotating posture change, should pay special attention to the choice of the ways of beam support, linear guide of deformation caused by the different ways may be dozens of times, greatly affect the stability and precision of the structure, and can also according to the deformation distribution shown in this graphic, In view of the large deformation area, the structural reinforcement and the tightening of the connection are adopted to reduce the deformation[7].Linear guide rail driven by beam support has the advantages of compact structure, flexible movement and spatial movement. In the design and use process, as long as the stability of the mechanism is well controlled in each link and the deformation is reduced, the function of the mechanism can be well played and the quality of the design and equipment can be improved.

\section{References}

1. Van-Cahn Tong;;Gung-ho Khim; Linear ball guide design optimization considering stiffness, friction force, and basic dynamic load rating using particle swarm optimization, Journal of Mechanical Science and Technology, 2020, 03

2. $\mathrm{Xu}$ Huawei, Cao Jangling, Nondestructive testing technology in the application of the electronic components failure analysis, Electronic technology and software engineering, 2019, 01

3. Song Xian chun, Fan Ming Zhen, Pre-tightening force on the roller More gesture guide pair of research on the effects of stiffness characteristics, Journal of Shandong construction university, 2019. 10

4. Zhang Shoo, Shu Qulin, Weibull distribution in the application of the linear rolling guide vice accuracy stable analysis, Group technology and production modernization, 2019. 03

5. Xu Haiwei, Cao Jiangping, Combined u-shaped pot body amplitude motion mechanism design, China Measurement \& Test, 2011,04.

6. Cao Jiangping, Filming for the tea of complete sets of equipment control system design, Graduation dissertation, university of electronic science and technology, 2013,12.

7. Cao Jiangping, Deformation analysis of rotating linear guide, ATDMAE 2020 Conference proceedings, 2020.08 